\title{
Clinical analysis of cardiac autonomic ganglion plexus ablation for bradyarrhythmia: Research protocol for an intervention study
}

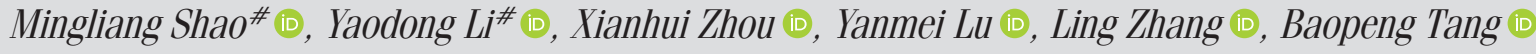

Department of Cardiac Pacing and Electrophysiology, Xinjiang Key Laboratory of Cardiac Electrophysiology and Remodeling, The First Affiliated Hospital of Xinjiang Medical University; Urumqi-China

\section{ABSTRACT}

Objective: This study aimed to explore the safety and effectiveness of selective cardiac autonomic ganglion plexus (GP) ablation on patients with bradyarrhythmia. The heart is controlled by its own intrinsic and central autonomic nerves. Increased cardiac vagal tone leads to sinus node dysfunction and atrioventricular conduction disorders, resulting in bradyarrhythmia. Pacemaker implantation can relieve the symptoms of arrhythmia caused by bradycardia, but it is not easy for patients to accept a pacemaker implantation as a form of treatment. Therefore, more and more attention has been paid to cardiac vagus nerve ablation.

Methods: In this study, 20 patients who met the inclusion criteria of GP ablation in the First Affiliated Hospital of Xinjiang Medical University from November 2019 to June 2020 were enrolled. Biochemical and other related examinations along with electrophysiological examinations were conducted before ablation, and then cardiac GP ablation was performed. The patients were followed up 3 times at 3, 6, and 12 months after the operation. Results: The minimum HR and mean HR were significantly increased after treatment with cardiac autonomic GP ablation ( $p<0.01)$. Moreover, the SDNN (Standard deviation of Normal-to-Normal Intervals) and RMSSD (Root mean square successive differences between successive $\mathrm{R}-\mathrm{R}$ intervals) was significantly decreased after treatment with cardiac autonomic ganglion plexus ablation for 6 months and 12 months ( $\mathrm{p}<0.01$ ). Conclusion: Cardiac GP ablation is relatively simple and easy to implement in units that have performed radiofrequency ablation for bradyarrhythmias. This procedure can be performed without any new equipment. Some patients with bradycardia may not have a permanent pacemaker implantation and may go in for additional treatment options.

Keywords: bradyarrhythmia, cardiac ganglionated plexus, ablation

Cite this article as: Shao M, Li Y, Zhou X, Lu Y, Zhang L, Tang B. Clinical analysis of cardiac autonomic ganglion plexus ablation for bradyarrhythmia: Research protocol for an intervention study. Anatol J Cardiol 2021; 25: 491-5.

\section{Introduction}

The development of neuroanatomy, neurophysiology, and functional imaging technology provides a new perspective for the central and peripheral mechanisms of the neural control of cardiac function (1). The heart is innervated by its own intrinsic and centrally derived autonomic nerves, of which the autonomic nerve called ganglion plexus (GP) is mainly distributed in the fat pad of the left atrial surface and the Marshall ligament of the right atrial surface. In addition, is the autonomic nerve is also distributed near the left atrium. The autonomic nervous system (ANS) inherent in the atrium receives signals from the central ANS but regulates cardiac functions, including autonomy, contractility, and conduction, independently. The autonomic GP contains afferent neurons from the atrial muscle and central ANS; efferent cholinergic and adrenergic neurons, which largely distribute in the aortic muscles of the pulmonary veins and the atrial muscle surrounding the GP; and a large number of connected neurons. There are extensive neural network connections between the GP and the myocardium and between the GP and the myocardium around the pulmonary veins. GP may also be associated with pericardium.

\footnotetext{
\#Contributed equally.
}

Address for Correspondence: Baopeng Tang, MD, Department of Cardiac Pacing and Electrophysiology, Xinjiang Key Laboratory of Cardiac Electrophysiology and Remodeling,

The First Affiliated Hospital of Xinjiang Medical University, No.137, Liyushan Road, 830054, Urumqi-China

Phone: 0991-4362658 E-mail: tangbaopeng@tom.com

Accepted Date: 02.02.2021 Available Online Date: 23.05.2021

(C) Copyright 2021 by Turkish Society of Cardiology - Available online at www.anatoljcardiol.com DOI:10.5152/AnatolJCardiol.2021.94797 


\section{HIGHLIGHTS}

- Cardiac ganglion plexus (GP) ablation is relatively simple and easy to implement in units that have performed radiofrequency ablation for bradyarrhythmias.

- Cardiac GP ablation can be carried out without any new equipment.

- Cardiac GP ablation is an additional treatment option for some patients with bradycardia who do not have a permanent pacemaker implantation.

The autonomic GP on the surface of the left atrium is mainly distributed in the left superior pulmonary vein, in the anterior (outside) of the top right superior pulmonary vein, along the Marshall ligament, outside the posterior left lower pulmonary vein, and outside the lower right pulmonary vein. Moreover, GP between the superior vena cava, aorta, and right pulmonary vein is considered a "Head Station" that is derived from the connection between the autonomic nerve fibers of the central body and is inherent in the left atrium. Specific part of GP in the left atrium can be determined by high frequency stimulation (HFS) with a frequency of $20-50 \mathrm{~Hz}$. The front degree is $10-15 \mathrm{~V}$, the pulse width is up to $10 \mathrm{~ms}$, and each stimulation lasts at least $5 \mathrm{~s}$. HFS caused by sinus arrest, sinus bradycardia, atrioventricular node block, and even hypotension and other vagus reflexes suggests the presence of GP. In vivo studies of humans have found that there are $4 \mathrm{GPs}$ on the left atrium of humans detected by HFS of the intima. In the animal model of vasovagal reflex, selective ablation of cardiac GP can weaken the control of parasympathetic nerves and significantly reduce cardiac inhibition response (2).

Similarly, the increase of vagal tone in human heart can reduce the excitability (3), autonomy, and conductivity of the myocardium, leading to functional abnormalities of the sinus node and atrioventricular conduction disorders, causing bradyarrhythmias, which includes intermittent high atrioventricular block, functional sinus node dysfunction, neurocardiogenic syncope, and so on. The heart structure and function in patients with increased vagal tone may be normal, but they often feel dizziness, syncope, palpitations, fatigue, chest tightness, shortness of breath, and other symptoms. Moreover, many patients have recurrent syncope, which can lead to trauma and other conditions, seriously affecting the quality of life and bringing mental burden to patients and their families. The drugs acting on vagus nerve have systemic effects, and the long-term side effects are more difficult to tolerate. Pacemaker treatment can alleviate symptoms, but it cannot solve the etiology. In the lifetime, patients need to change the pacemaker many times, which increases economic and psychological burden as well as a variety of complications. Most patients are young and find it difficult to accept pacemaker implantation as a form of treatment. Therefore, for the slow heart rate caused by increased vagal tone, cardiac GP ablation has attracted more and more attention, and rapid progress has been observed in clinical practice (4).

\section{Methods}

\section{Subjects}

In this study, 20 patients who met the inclusion criteria of GP ablation in the First Affiliated Hospital of Xinjiang Medical University from November 2019 to June 2020 were enrolled. Inclusion criteria included the following: (1) patients with symptoms of dizziness, apopsychia, cardiopalmus, weakness, chest distress, and shortage of breath, which were not caused by high grade atrioventricular block and sick sinus syndrome and proved by electrogardiogram (ECG) or holter; (2) patients who had the characteristics that showed that permanent pacemaker implantation was needed; (3) patients with positive atropine test for esophageal pacing; and (4) patients aged 18 to 50 years. Exclusion criteria included the following: (1) patients with welldiagnosed sinus node lesions and atrioventricular heart block, hypertrophic cardiomyopathy, pulmonary hypertension, epilepsy, transient ischemic attack, and subclavian vein theft syndrome; (2) patients with serious complications, including myocardial infarction, New York Heart Association heart function III-IV, diabetes, and incurable diseases; and (3) patients who had undergone cardiac surgery, catheter ablation, and pace maker implantation in the past.

\section{Examination before ablation}

In this study, biochemistry examination of GAP43 (growth associated protein-43), TH (tyrosine hydroxylase), and highsensitivity C-reactive protein; blood routine examination; urine routine examination; stool routine examination; and ECG, echocardiogram, dynamic electrocardiogram, and esophageal pacing examination were performed on patients.

\section{Electrophysiological examination}

Electrophysiological examination was carried out before and after ablation therapy to evaluate the functions of sinoatrial node and atrioventricular node. Determination of sinus node recovery time, venturi blockade point, atrioventricular conduction time, $\mathrm{AH}$ and $\mathrm{HV}$ intervals as well as changes before and after medication and ablation were also carried out.

\section{GP ablation}

For patients with bradyarrhythmia, understanding that the real cause is the malfunction of vagus node rather than organic lesion is important for a successful treatment with ablation.

\section{Localization methods}

Although the distribution of atrial GP is relatively concentrated, there are still great differences among different individuals. In recent years, 3 localization methods have been applied in the study of GP, including HFS, spectral analysis (SA), and anatomical localization (AA). Research has shown that several GP localization methods are often complementary to each other, and there are relatively few studies on the application of a single localization method. Since the earliest animal experiments, HFS has been used as a way to locate GP (5-7). The advantage of HFS 
is that it can clearly find out the distribution area of GP, and it has good accuracy and repeatability; therefore, it is now being widely used. The disadvantage of HFS is that it can stimulate the vagus nerve that easily causes discomfort in patients. The higher the stimulation intensity, the stronger the discomfort. Discomfort also tends to cause changes in autonomic nervous tone, which may potentially affect the endpoint of ablation. Repeated stimulation of the atrium may induce a variety of arrhythmias, including atrial fibrillation, atrial flutter, and so on (8).

SA was first discussed by Pachon et al. (9) in 2004. Using a special calculation program and a 32-channel multi-conducting physiological recorder (pachon-teb2002), the electrical signals in the cavity are transformed from the time domain to the frequency domain by fast Fourier transform (FFT), thereby analyzing the composition of the electrical signals in the cavity more directly (9). The traditional electrophysiological recorder can only record the amplitude of the electrical signals in the cavity with time. However, after FFT processing, the original waveform is processed into a visual spectrum in the frequency domain, that is, a collection of multiple sinusoidal waves. After different filter settings in the process of recording, the automatic analysis of potential components can be realized. Pachon et al. (9) successfully recorded the different spectral characteristics of atrial potential by 3 commonly used bandpass filters, including bandpass filters with frequencies $30-500 \mathrm{~Hz}, 100-500 \mathrm{~Hz}$, and $300-500$ $\mathrm{Hz}$. Pachon et al. (9) believed that according to the different spectral characteristics of atrial potential, it can be divided into typical myocardial tissue, which is characterized by homogeneity, fast conduction, and relatively left shift of the spectrum and nerve fibers with heterogeneous, highly fragmented, and rightshifted distribution. The distribution area of GP can be identified by SA of the potential in the cavity. Compared with HFS, SA causes less pain in patients and has a certain degree of accuracy (10).

However, there are some disadvantages of SA. SA needs to use a special electrophysiological recorder and supporting software to map the entire atrium, which is more time consuming. Therefore, AA is often referred to, and the accuracy has not been widely confirmed. Currently, SA is only used to assist other localization methods such as HFS, that is, mapping was performed near the normal anatomical site of GP or verified in the HFS positive area.

With the appliance of localization methods such as HFS and SA, the understanding of the distribution area of GP has gradually improved, and more and more researchers have begun to adopt AA (sometimes supplemented by other localization methods) for empirical ablation. The 3-dimensional electrophysiology (EP) navigation system was used to model the atrium, and the location of GP was preliminarily determined according to the anatomical structure of the model. The advantage of $A A$ is that it does not require mapping, saving time, low requirements for equipment, and less pain for patients, whereas the disadvantage is that depending on the empirical ablation of $A A$, it may increase unnecessary melting point, causing more intra-atrial scar tissue, increasing risk of future atrial arrhythmia and complications.
The 3 commonly used positioning methods are often applied in combination because of their advantages and disadvantages. However, we still need a localization approach or strategy that strikes a better balance between accuracy, convenience, and security.

\section{Ablation method}

After locating the cardiac ganglia, use the $3.5 \mathrm{~mm}$ ablation electrode to use EP shuttle (Johnson\&Johnson, USA) temperature-controlled radiofrequency ablation under the guidance of the three-dimensional mapping system. The upper limit of power is less than $45 \mathrm{~W}$ and the upper limit of temperature is not higher than $40^{\circ} \mathrm{C}$. The ablation is performed in sequence according to LSGP, RAGP, LIGP, and RIGP, and the ablated part is marked on the three-dimensional model of the left atrium immediately. $A$ rapid drop in blood pressure, transient ventricular arrest, at least $50 \%$ prolongation of the RR interval, or atrioventricular block caused by high-frequency stimulation or ablation is defined as a response to the vagus response. Confirm that the site is an effective GP stimulation site point. All ablation sites need to be ablated until the vagus reaction disappears, and each discharge is greater than $60 \mathrm{~s}$.

\section{End of ablation}

The increased sinus rhythm, disappearance of vagus reflex, and completion of anatomical ablation suggested the end of ablation. The end of ablation required the disappearance of abnormal electrophysiological signals, increased sinus rhythm, or increased Wenckebach point of atrioventricular conduction (Fig. 1-4).

1. Observation and follow up: Baseline observation indicators. The general clinical data of patients, including demographic features, physical checkup, macrobiochemistry, blood routine examination, urine routine examination, stool routine examination, electrocardiogram, echocardiogram, dynamic electrocardiogram, esophageal pacing examination, and present medication were collected.

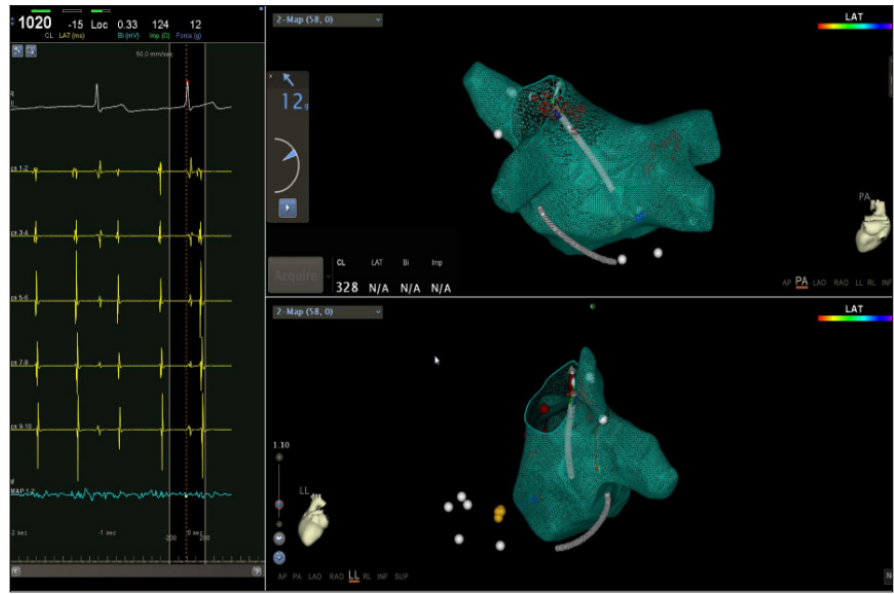

Figure 1. Ablation at the top of the left lung showed bradycardia, affecting the vagal ganglion plexus 


\begin{tabular}{|c|c|c|c|c|c|}
\hline Items & Od & 1d & $3 m$ & $6 m$ & $12 m$ \\
\hline Max HR & $117.2 \pm 20.7$ & $123.6 \pm 13.9$ & $123.8 \pm 12.8$ & $121.6 \pm 12.5$ & $121.4 \pm 12.5$ \\
\hline Mean HR & $66 \pm 11.98$ & $77.9 \pm 6.3$ & $72.2 \pm 2.8^{*}$ & $72.2 \pm 2.8^{*}$ & $72.2 \pm 2.3^{*}$ \\
\hline SDNN (ms) & $131.9 \pm 65.0$ & $99.13 \pm 31.34$ & $102.19 \pm 26.769$ & $105.74 \pm 23.80^{*}$ & $106.30 \pm 20.73^{*}$ \\
\hline
\end{tabular}

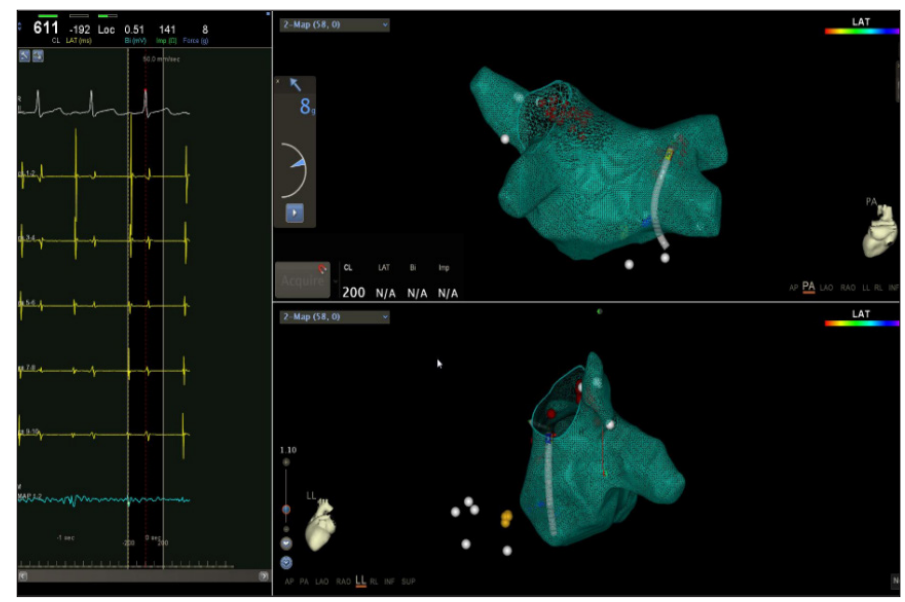

Figure 2. Ablation at the top of the right upper lung showed accelerated heart rate, affecting the vagal ganglion plexus

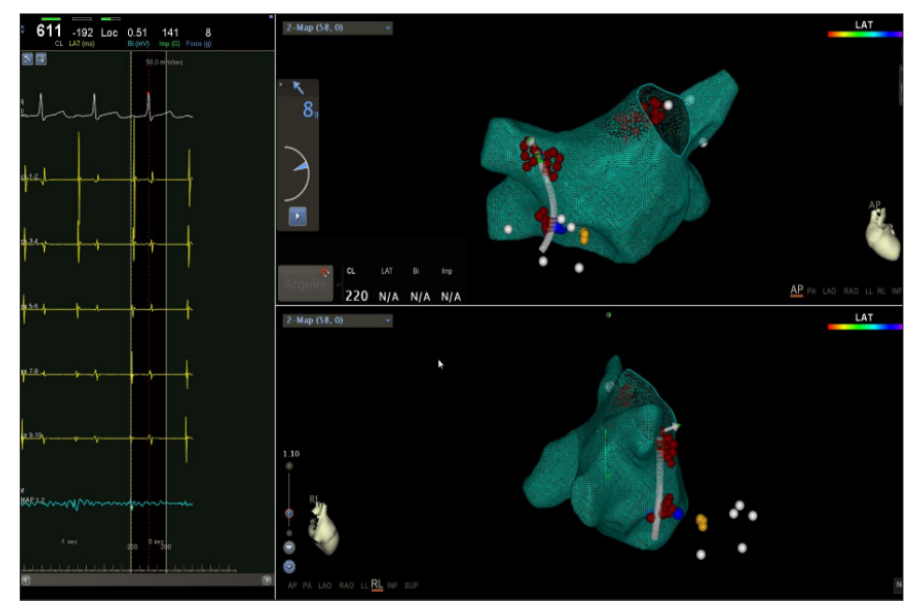

Figure 3. Ablation at the top of the right upper lung showed accelerated heart rate, affecting the vagal ganglion plexus

2. Follow-up indicators: The patients were followed up 3 times at 3,6 , and 12 months after the operation. During every follow up, echocardiography, 12-leads ECG, 24-hours ambulatory ECG, and transesophageal atrial pacing were performed. During the first follow up, pulmonary vein computed tomography angiography was added.

\section{Statistical analysis}

The measurement data were expressed as means \pm stan-

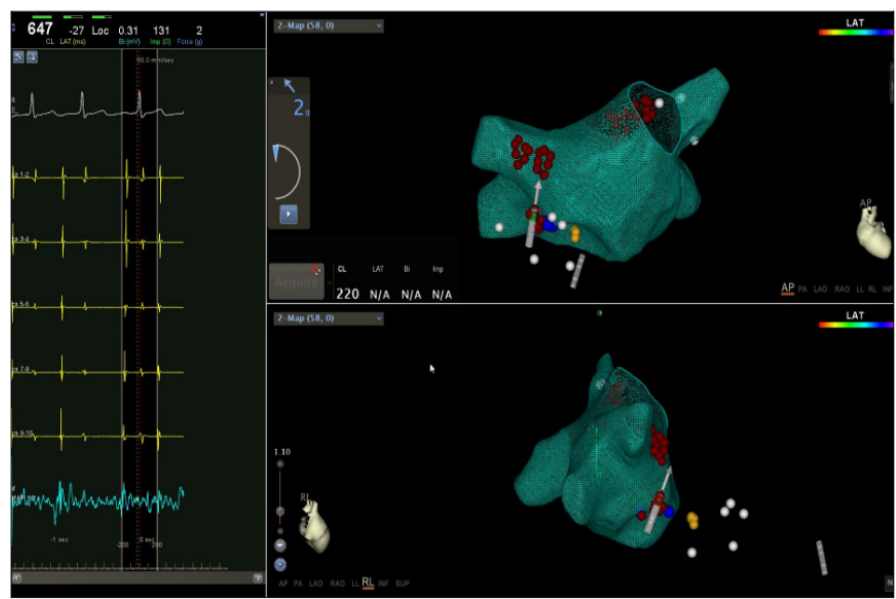

Figure 4. Ablation of fossa ovale resulted in accelerated heart rate, which affected the vagal ganglion plexus

dard deviation and compared by Normality test and repeated measures analysis of variance. Differences were considered statistically significant at $p<0.05$.

\section{Results}

As shown in Table 1, the changes of maximum heart rate (HR) after treatment were not significantly different in comparison with those before treatment ( $>0.05)$. However, the minimum HR and mean $\mathrm{HR}$ were significantly increased after treatment with cardiac autonomic ganglion plexus GP ablation $(p<0.01)$. Moreover, the SDNN (standard deviation of normal-to-normal intervals) and RMSSD (root mean square successive differences between successive $R-R$ intervals) was significantly decreased after treatment with cardiac autonomic ganglion plexus ablation for 6 months and 12 months $(p<0.01)$.

\section{Discussion}

Bradyarrhythmia mediated by vagus nerve are common in clinical practice. Most of the patients with bradyarrhythmia are young and have severe symptoms with normal cardiac structure and function. The side effect of drug therapy is more, and the treatment effect is not good. Pacemaker replacement is required for pacing therapy, which is not a radical treatment and is diffi- 
cult for patients to receive. The concept of ablation and the development of ablation techniques provide a new therapeutic method for these patients. The effector fibers of vagus nerve are mainly distributed around the sinoatrial node, atrioventricular node, and posterior wall of atrium, and their abnormal function can cause bradyarrhythmias. Vagal nerve fibers are mainly distributed in the ventricle and sinus, and they can cause vasovagal reflex. Despite the repeatability and good clinical results, a large number of patients need to be treated and followed up to reach definitive conclusions.

However, the follow-up period of present relevant studies is only 1 year, which cannot reflect the long-term effect of GP ablation. Considering that it is difficult to achieve complete denervation of GP by endocardial ablation, it is not clear whether there is a long-term nerve regeneration. A phenomenon of nerve regeneration after sympathetic nerve removal by surgical maze surgery has been reported, suggesting that the regeneration of ANS may affect the long-term effect of GP ablation. Moreover, the sample size in this study was also small. The results of this study should be further investigated.

\section{Study limitations}

At present, the target location of GP ablation and the determination of the ablation end point are not very accurate. The technical means to identify nerve potentials still need to be accurately displayed by the technological advancement of ganglion marker scanning, MRI and other imaging aids. At the same time, the follow-up time span after ablation is long. There are a large number of external factors in the patient's long-term living environment. The objective risk is difficult to monitor and eliminate in real time. The multi-factor superimposed compound effect, to a certain extent, interferes with the direction of GP ablation in the treatment of bradycardia. The clinical efficacy study.

\section{Conclusions}

According to the above-mentioned clinical research results, left atrial endometrial GP ablation can increase sinus heart rate and relieve discomfort in patients with symptomatic bradyarrhythmia. There is a process in the treatment of diseases with more complications such as bradyarrhythmia. Medium, safe and effective, it is a treatment for bradyarrhythmia with high potential. The long-term benefits of this treatment strategy require randomized and controlled studies on the basis of larger samples to ensure that the conclusions are in-depth confirmed.

Ethics and dissemination: The protocol is approved by the Institutional Review Board of the First Affiliated Hospital of Xinjiang Medical University (Approval number: K201910-02). The results will be published in peer-reviewed journals and communicated in cardiovascular conferences.

Trial status: The trial was registered in October, 2019 (Chinese Clinical Trial Registry, ChiCTR1900027305). The completion of this study is expected in February 2020. Chinese Clinical Trial Registry, ChiCTR1900027305. Registered on 8 November 2019. chictr.org.cn.

Institutional and financial support: The authors thank from National Natural Science Foundation of China (No.81873487) for the financial support.

Acknowledgments: Thank the staff of the catheterization room and ECG room of the First Affiliated Hospital of Xinjiang Medical University for their support to this study, and thank the selected patients for their cooperation in this study.

\section{Conflict of interest: None declared.}

Peer-review: Internally peer-reviewed.

Author contributions: Concept - M.S., Y.Li; Design - M.S., Y.Li; Supervision - M.S., Y.Li; Fundings - X.Z., Y.Lu; Materials - X.Z., Y.Lu; Data collection \&/or processing - L.Z., B.T.; Analysis \&/or interpretation - L.Z., B.T.; Literature search - M.S., Y.Li; Writing - L.Z., B.T.; Critical review - M.S., Y.Li, X.Z., Y.Lu, L.Z., B.T.

\section{References}

1. Palma JA, Benarroch EE. Neural control of the heart: recent concepts and clinical correlations. Neurology 2014; 83: 261-71. [Crossref]

2. Wang X, Luo D, Liu S, Ou W, Ma R, Yu X, et al. Selective ablation of atrial ganglionated plexus attenuates vasovagal reflex in a canine model. Pacing Clin Electrophysiol 2019; 42: 13-9. [Crossref]

3. Gourine AV, Ackland GL. Cardiac Vagus and Exercise. Physiology (Bethesda) 2019; 34: 71-80. [Crossref]

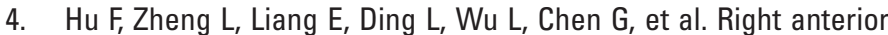
ganglionated plexus: The primary target of cardioneuroablation? Heart Rhythm 2019; 16: 1545-51. [Crossref]

5. Yao Y, Shi R, Wong T, Zheng L, Chen W, Yang L, et al. Endocardial autonomic denervation of the left atrium to treat vasovagal syncope: an early experience in humans. Circ Arrhythm Electrophysiol 2012; 5: 279-86. [Crossref]

6. Liang Z, Jiayou Z, Zonggui W, Dening L. Selective atrial vagal denervation guided by evoked vagal reflex to treat refractory vasovagal syncope. Pacing Clin Electrophysiol 2012; 35: e214-8. [Crossref]

7. Rivarola E, Hardy C, Sosa E, Hachul D, Furlan V, Raimundi F, et al. Selective atrial vagal denervation guided by spectral mapping to treat advanced atrioventricular block. Europace 2016; 18: 445-9. [Crossref]

8. Aksu T, Golcuk E, Yalin K, Guler TE, Erden I. Simplified Cardioneuroablation in the Treatment of Reflex Syncope, Functional AV Block, and Sinus Node Dysfunction. Pacing Clin Electrophysiol 2016; 39: 42-53. [Crossref]

9. Pachon MJC, Pachon MEI, Pachon MJC, Lobo TJ, Pachon MZ, Vargas RN, et al. A new treatment for atrial fibrillation based on spectral analysis to guide the catheter RF-ablation. Europace 2004; 6: 590-601. [Crossref]

10. Rivarola EW, Hachul D, Wu T, Pisani C, Hardy C, Raimundi F, et al. Targets and End Points in Cardiac Autonomic Denervation Procedures. Circ Arrhythm Electrophysiol 2017; 10: e004638. [Crossref] 